\title{
Sparsity Aware Multiuser Detection for Machine to Machine Communication
}

\author{
Fabian Monsees, Carsten Bockelmann, Dirk Wübben and Armin Dekorsy \\ Department of Communications Engineering \\ University of Bremen, Germany \\ E-mail: \{monsees,bockelmann,wuebben,dekorsy\}@ ant.uni-bremen.de
}

\begin{abstract}
With the expected growth of Machine-to-Machine (M2M) communication, new requirements for future communication systems have to be considered. Traffic patterns in M2M communication fundamentally differ from human based communication. Especially packets in M2M are rather small and transmitted sporadically only. Moreover, nodes for M2M communication are often of reduced functionality which makes complex control overhead or resource management infeasible for such devices. Assuming a star-topology with a central aggregation node that processes all node information one possibility to reduce control signaling is to shift the activity detection fully to the central aggregation node. The methodology of a joint activity and data detection differs strongly from common communication scenarios since errors during the activity detection are fundamentally different from errors made at data detection. In this paper we introduce a non-linear joint activity and data detector for M2M communication. The performance regarding data and activity errors is assessed and compared to a scenario where node activity is known by the aggregation node.
\end{abstract}

\section{INTRODUCTION}

Machine-to-Machine (M2M) communication is expected to grow tremendously in the next years [1], thereby posing new challenges for both existing communication systems, designed with human communication in mind, and for existing M2M solutions alike. On the one hand, standards like LTE have to be extended to cope with the requirements of M2M communication, which are often differing strongly from requirements for speech communication and human data access. For example, management overhead like mobility management or sophisticated resource allocation is not well suited for lowrate communication of sensor nodes that are only transmitting sporadically. In the current frame structure of LTE-A, at most a few tens of M2M devices can be supported with control channel elements (CCEs) allocated within a subframe [2]. This is far below the target number of M2M devices envisioned for the future. On the other hand, a growing number of nodes will be challenging for existing M2M standards like the IEEE standards 802.15.4 and 802.15.1. Both are based on Carrier Sense Multiple Access with Collision Avoidance (CSMA/CA) and spread spectrum techniques. A thorough overview of current activities in different standardization bodies like ETSI and 3GPP can be found in [1]. Furthermore, detailed requirements on future M2M systems and an assessment of current technologies can be found in the deliverable D2.1 of the European project EXALTED [3].
In order to efficiently cope with a high number of sensor nodes, which are expected to only sporadically transmit small packets with low data rates, new approaches on the physical layer are required. Major concerns are low control signal overhead and improved power efficiency, for long battery life of low-cost sensors.

Within this work, we focus on physical layer aspects of M2M. We state that signaling overhead can significantly be reduced by avoiding any control signaling regarding the activity of sensor nodes prior to transmission. A promising approach is to extend the receivers with the capability to detect activity of the nodes. In consequence, the receiver has to perform joint activity and data detection.

To point out the main idea, we consider a star topology, where sensors desire to transmit data to a central aggregation node as done in industrial applications such as smart factories, medical applications or logistics [4]. We further assume a random channel access facilitated by Code Division Multiple Access (CDMA) which is very attractive for M2M scenarios, due to its adaptive and flexible support of different data rates, as well as Qualities of Service, and flexible and scalable number of supported devices. The existence of a central aggregation node allows for high processing power and sophisticated Multi-User detection. Due to the assumption of sporadic transmission, only a small number of sensors are active at any given time. Therefore, the Multi-User signal composed of signals from all sensors in the network is sparse, i.e., the information is contained in a few signal components only.

Knowledge about the node activity can be exploited by the detector which we call Sparsity Aware detection. Consequently, control signaling concerning node activity can be lessoned by moving the activity detection problem fully to the central aggregation node which performs joint activity and data detection for the nodes. In this paper we focus on non-linear Sparsity Aware Multi-User detection facilitated by the application of a Maximum a Posteriori detector. We consider the detection at symbol rate which requires the application of a pre-whitening filter to ensure decorrelated white Gaussian noise at the input of our detector. Moreover, the framework of a joint activity and data detection necessitates different error handling compared to systems where only data detection is performed. Beside the wrong detection of symbols at the data detection, the activity of a 


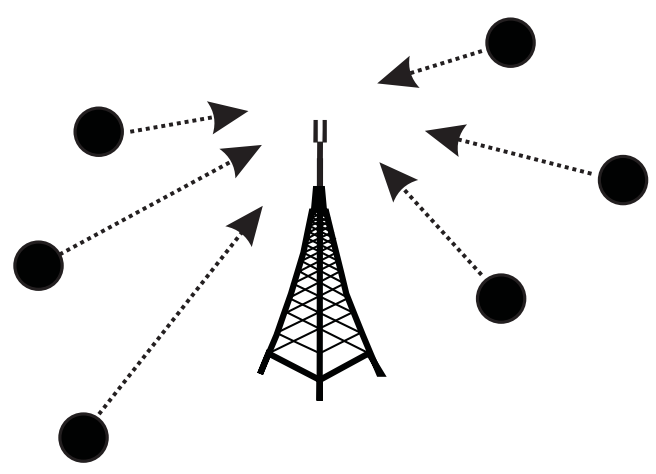

Fig. 1. Sensor network - star topology

node can be estimated wrongly as well. This class of error is fundamentally different from those made at symbol detection. For example, if an active node is estimated as inactive, the data of this node is lost. Contrary, inactive nodes that are wrongly estimated to be active can possibly be identified by higher layer error handling applications such as check codes that identify invalid pieces of information like invalid frames. To capture the performance of the detector appropriately, we introduce new error classes on symbol and frame basis that allow to asses the performance of the detector regarding joint activity and data detection. To this end, we consider the application of a Sparsity Aware Multi User-Detector which we apply to the symbol rate model of the Multi-User-CDMA (MU-CDMA) system for the joint activity and data detection and evaluate the performance according to the aforementioned error classes. We therefore investigate the occurrence of the error events on symbol level and on frame level.

\section{SYSTEM MODEL}

\section{A. Machine-to-Machine Scenario}

In this paper, we consider a M2M scenario, where $K$ sensor nodes communicate with a central aggregation node, as shown in Fig. 1, typically denoted as a star topology. All sensor nodes are devices of low complexity, while the central node allows for advanced signal processing, such as sophisticated detection and message aggregation for forwarding. In principle, the sensor nodes are active on occasion only, i.e., measurements have to be transmitted event driven, which leads to sporadic communication. In contrast, the aggregation node is active all the time to process incoming messages. To model sensor node activity, we adopt a statistical approach, where each sensor node is active for a short time period, with a given activity probability $p_{a}$. This activity probability, which we assume to be identical for all sensor nodes, determines the number of active nodes in a statistical sense. In the following, the base assumption is that out of the $K$ sensor nodes in the network the majority are silent, i.e., the activity probability is rather small, with $p_{a} \ll 1$.

To facilitate sporadic and simultaneous medium access, CDMA with Pseudo random Noise (PN) sequences is used instead of the CSMA/CA scheme employed in IEEE standards. Here, we choose PN sequences due to their well known correlation properties for uplink communication [5]. CDMA offers a number of attractive properties for $\mathrm{M} 2 \mathrm{M}$ communication as noted before. It allows for a very flexible non-orthogonal medium access with some degree of asynchronicity on the chip level between sensor nodes.

\section{B. CDMA Symbol-rate System Model}

We assume that a node $k, 1 \leq k \leq K$ is active with probability $p_{a}$ and transmits a frame containing $L_{k}$ symbols from the modulation alphabet $\mathcal{A}$. With CDMA, these symbols are spread to chips by a node specific spreading sequence of length $N$. After spreading the active nodes transmit the chips over a frequency selective Rayleigh fading channel with impulse response length of $L_{h, k}$ chips where the channel coefficients $h_{k, n}, 1 \leq n \leq L_{h, k}$ obey the same uncorrelated Rayleigh fading statistics with zero mean and variance $1 / L_{h, k}$. Without loss of generality, we assume that $L_{k}=L \forall k$ and $L_{h, k}=L_{h} \forall k$. This assumption allows to model the node specific spreading and convolution with the underlying channel by the matrix $\mathbf{A} \in \mathbb{R}^{N+L_{h}-1 \times K}$. This consideration simplifies the formulation of a matched-filter that we assume to be applied at the aggregation node in order to go from chip rate back to symbol rate. Moreover, we assume $L_{h}<N$ such that inter-symbol interference can be ignored and multi-user interference becomes the main performance limiting factor of the CDMA system [6][5]. Thus, we capture the symbol clock input output relation of our system as

$$
\mathbf{y}_{i}=\mathbf{A}^{T} \mathbf{A} \mathbf{x}_{i}+\mathbf{A}^{T} \mathbf{w} .
$$

The vector $\mathbf{x}_{i}, 1 \leq i \leq L$ contains the symbols from all $K$ nodes at the time instance $i$. To incorporate node inactivity, we model an inactive node to transmit only the zero symbol and we have $x_{k}=0$ for inactive nodes and $x_{k} \in \mathcal{A}$ for active nodes. The Modulation alphabet $\mathcal{A}$ can be any real valued alphabet such as Amplitude Shift Keying (ASK) or Binary Phase Shift Keying (BPSK). The assumption of a real valued alphabet is not a general restriction. The methods applied in this paper can be used for any complex system using the equivalent real valued description. The elements of the source vector $\mathbf{x}_{i}$, consequently, belong to the augmented symbol alphabet $\mathcal{A}_{0}=\mathcal{A} \cup 0$. With this model and a node activity probability of $p_{a}$, the $k$ th element of $\mathbf{x}_{i}$ contains a zero with probability $1-p_{a}$. We further model node activity on a frame basis, i.e., the node $k$ is either active and transmits a frame containing $L$ symbols from $\mathcal{A}$ or the node is silent and is modeled to transmit a whole frame containing only zeros. At the aggregation node, matched filtering is done by simply collecting $N+L_{h}-1$ chips which are filtered by $\mathbf{A}^{T}$. The chip rate noise vector $\mathbf{w}$ contains uncorrelated white Gaussian noise samples with covariance matrix $\mathrm{E}\left(\mathbf{w w}^{T}\right)=\sigma_{n}^{2} \mathbf{I}_{N+L_{h}-1}$. Equation (1) clearly shows that the noise is filtered by the matched filter leading to correlated noise at symbol rate with covariance matrix

$$
\tilde{\boldsymbol{\Phi}}=\mathrm{E}\left(\mathbf{A}^{T} \mathbf{w} \mathbf{w}^{T} \mathbf{A}\right)=\sigma_{n}^{2} \mathbf{A}^{T} \mathbf{A} .
$$


Additionally, we assume that the number of nodes is lower than the length of the spreading sequence $K \leq N$ which corresponds to a CDMA system load of $\beta=K / N \leq 1$.

\section{Noise Pre-Whitening}

We apply non-linear detection for the Multi-User system described by (1). These detector assumes the noise to be uncorrelated, we therefore introduce two pre-whitening schemes that can be applied to decorrelate the noise. The goal of a pre-whitening filter $\mathbf{P}$ is to diagonalize the noise covariance matrix at symbol rate (2). Consequently, we have to find $\mathbf{P}$ such that

$$
\mathbf{\Phi}=\mathbf{P} \tilde{\mathbf{\Phi}} \mathbf{P}^{T} \stackrel{!}{=} \sigma_{n}^{2} \mathbf{I}_{K}
$$

is fulfilled, with $\mathbf{I}_{K}$ being the identity matrix of dimension $K$. Within this work we consider two different concepts for the formulation of the pre-whitening filter.

1) Eigenvalue Based Pre-Whitening: Given the noise covariance matrix at symbol rate from (2), leads after Eigen Value Decomposition (EVD) of the matrix $\tilde{\boldsymbol{\Phi}}$ to

$$
\tilde{\boldsymbol{\Phi}}=\sigma_{n}^{2} \mathbf{V} \boldsymbol{\Sigma} \mathbf{V}^{T}
$$

With (3) we have

$$
\mathbf{\Phi}=\sigma_{n}^{2} \mathbf{P} \mathbf{V} \boldsymbol{\Sigma} \mathbf{V}^{T} \mathbf{P}^{T},
$$

and if $\mathbf{P}=\boldsymbol{\Sigma}^{-1 / 2} \mathbf{V}^{T}$, (5) is reduced to

$$
\begin{aligned}
\boldsymbol{\Phi} & =\sigma_{n}^{2} \boldsymbol{\Sigma}^{-1 / 2} \mathbf{V}^{T} \mathbf{V} \boldsymbol{\Sigma} \mathbf{V}^{T} \mathbf{V} \boldsymbol{\Sigma}^{-1 / 2} \\
& =\sigma_{n}^{2} \mathbf{I}_{K} .
\end{aligned}
$$

This technique is strongly connected to the Karhunen-Loève Transformation and sometimes denoted as Discrete KarhunenLoève Transform (D-KLT) [7].

2) Cholesky Decomposition: Another possibility for diagonalizing the noise covariance matrix (2) is to apply the skinny QR decomposition of the matrix A [8, p. 217] which leads to $\mathbf{A}=\mathbf{Q R}$ with $\mathbf{Q} \in \mathbb{R}^{N+L_{h}-1 \times K}$ and $\mathbf{R} \in \mathbb{R}^{K \times K}$ and $\mathbf{Q}$ is column orthonormal such that $\mathbf{Q}^{T} \mathbf{Q}=\mathbf{I}_{K}$ holds. Consequently, (2) can be decomposed to

$$
\begin{aligned}
\tilde{\boldsymbol{\Phi}} & =\sigma_{n}^{2} \mathbf{R}^{T} \mathbf{Q}^{T} \mathbf{Q R} \\
& =\sigma_{n}^{2} \mathbf{R}^{T} \mathbf{R},
\end{aligned}
$$

where $\mathbf{R}^{T}$ is the lower triangular Cholesky factor of the positive definite matrix product $\mathbf{A}^{T} \mathbf{A}$. The covariance matrix (3) simplifies by choosing $\mathbf{P}=\mathbf{R}^{-H}$ to

$$
\begin{aligned}
\tilde{\boldsymbol{\Phi}} & =\sigma_{n}^{2} \mathbf{R}^{-H} \mathbf{R}^{T} \mathbf{R} \mathbf{R}^{-1} \\
& =\sigma_{n}^{2} \mathbf{I}_{K} .
\end{aligned}
$$

As the matrix $\mathbf{R}^{T}$ and its inverse are both lower triangular, this methodology is preferable for implementation since the filtering with $\mathbf{P}$ is not as costly compared to the multiplication with a non upper triangular matrix as for the D-KLT [9]. We further note that the input-output relation for both prewhitening filter concepts is bijective which concludes that both pre-whitening filters do not lead to an information loss after filtering.

\section{Sparsity Aware Sphere Detector (SA-SD)}

The main task of our detector is to recover the symbols of all nodes from the Multi-User signal described by (1). With the application of a pre-whitening filter we reformulate the input output relation of our system to

$$
\begin{aligned}
\mathbf{y}_{i} & =\mathbf{P} \mathbf{A}^{T} \mathbf{A} \mathbf{x}_{i}+\mathbf{P} \mathbf{A}^{T} \mathbf{w} \\
& =\mathbf{T} \mathbf{x}_{i}+\tilde{\mathbf{w}}
\end{aligned}
$$

Here the matrix $\mathbf{T} \in \mathbb{R}^{K \times K}$ corresponds to effective channel matrix incorporating spreading, convolution with the channel, de-spreading and pre-whitening. The vector $\tilde{\mathbf{w}}$ represents the white and uncorrelated noise at the output of the pre-whitening filter. With (9), we see that the goal is to recover a vector that contains with high probability more zeros than elements from $\mathcal{A}$. A vector with only a few element that are non-zero is called a sparse vector. The sparsity of a vector is defined as the number of elements that are non-zero and is mathematically expressed by the zero-pseudo norm $S_{x}=\|\mathbf{x}\|_{0}$. In our case we assume that the aggregation node has knowledge about $p_{a}$ but neither about the particular sparsity of $\mathbf{x}_{i}$ nor the position of unequal zero symbols. All in all, the aggregation node has statistical knowledge about the sparsity of $\mathbf{x}_{i}$.

In [10] the authors showed that the Maximum a Posteriori (MAP) detector for the detection of a sparse vector that is corrupted by white Gaussian noise reads

$$
\hat{\mathbf{x}}=\min _{\mathbf{x}_{i} \in \mathcal{A}_{0}}\left\|\mathbf{y}_{i}-\mathbf{T} \mathbf{x}_{i}\right\|_{2}^{2}+2 \sigma_{n}^{2}\left\|\mathbf{x}_{i}\right\|_{0} \ln \left(\frac{1-p_{a}}{\left(p_{a} /|\mathcal{A}|\right)}\right) .
$$

Here $|\mathcal{A}|$ is the cardinality of the modulation alphabet used for data transmission. The optimization problem formulated in (10) can be interpreted as a least-squares problem that is regularized by a term reflecting the a priori probability of $\mathbf{x}_{i}$. The application of the zero-pseudo norm leads to costs that are increased by exactly $2 \sigma_{n}^{2} \ln \left(\frac{1-p_{a}}{p_{a} /|\mathcal{A}|}\right)$ for each element from the symbol alphabet $\mathcal{A}$ contained in the vector $\mathbf{x}_{i}$. Therefore, the regularization term forces the detector to prefer sparse solutions for $\mathbf{x}_{i}$ and scales with the noise power $\sigma_{n}^{2}$ making non-zero symbol detection in a low Signal-to-Noise (SNR) range costly.

The optimization problem stated in (10) is discrete over the augmented alphabet $\mathcal{A}_{0}$ and can efficiently be solved by a Sparsity Aware Sphere Detector (SA-SD). We reformulate the optimization problem by writing $\mathbf{T}=\mathbf{Q R}$ with $\mathbf{Q}$ being an unitary matrix, i.e., $\mathbf{Q}^{T} \mathbf{Q}=\mathbf{Q} \mathbf{Q}^{T}=\mathbf{I}_{K}$ and $\mathbf{R}$. We can rewrite (10) to

$$
\begin{aligned}
& \hat{\mathbf{x}}=\min _{\mathbf{x}_{i} \in \mathcal{A}_{0}}\left\|\mathbf{y}_{i}-\mathbf{Q R} \mathbf{x}_{i}\right\|_{2}^{2}+2 \sigma_{n}^{2}\left\|\mathbf{x}_{i}\right\|_{0} \ln \left(\frac{1-p_{a}}{\left(p_{a} /|\mathcal{A}|\right)}\right) \\
& \hat{\mathbf{x}}=\min _{\mathbf{x}_{i} \in \mathcal{A}_{0}}\left\|\mathbf{Q}^{T} \mathbf{y}_{i}-\mathbf{Q}^{T} \mathbf{Q R} \mathbf{x}_{i}\right\|_{2}^{2}+2 \sigma_{n}^{2}\left\|\mathbf{x}_{i}\right\|_{0} \ln \left(\frac{1-p_{a}}{\left(p_{a} /|\mathcal{A}|\right)}\right) \\
& \hat{\mathbf{x}}=\min _{\mathbf{x}_{i} \in \mathcal{A}_{0}}\left\|\tilde{\mathbf{y}}_{i}-\mathbf{R} \mathbf{x}_{i}\right\|_{2}^{2}+2 \sigma_{n}^{2}\left\|\mathbf{x}_{i}\right\|_{0} \ln \left(\frac{1-p_{a}}{\left(p_{a} /|\mathcal{A}|\right)}\right)
\end{aligned}
$$

Note that the regularization term in (11) is monotonically increasing in the number of symbols from $\mathcal{A}$ contained in 
$\mathbf{x}_{i}$ which allows for efficient implementation by a Sphere Detector [10][11].

Calculating the QR decomposition of $\mathbf{T}$ can be omitted by the application of the Cholesky Decomposition based prewhitening filter. In this case we can rewrite the matrix $\mathbf{T}$ by the application of the skinny QR decomposition. With (9) and $\mathbf{P}=\mathbf{R}^{-H}$ we have

$$
\begin{aligned}
\mathbf{T} & =\mathbf{P A}^{T} \mathbf{A} \\
& =\mathbf{P}(\mathbf{Q R})^{T} \mathbf{Q R} \\
& =\mathbf{R}^{-H} \mathbf{R}^{T} \mathbf{Q}^{T} \mathbf{Q R} \\
& =\mathbf{R} .
\end{aligned}
$$

This simplification does not hold for the concept of EVD based pre-whitening and makes the Cholesky decomposition based pre-whitening preferable for the application of a SphereDetector.

\section{A. Connection to Compressed Sensing}

The detection of a sparse vector from a noise corrupted measurement is strongly related to the field of Compressed Sensing (CS) [12][13]. In CS, a sparse vector of dimension $n$ can be recovered with high success rate even if the number of measurements $m$ is smaller than $n$, which requires to solve an under-determined set of equations. Even though the scenarios considered within this work are fully or even over-determined, CS theory was shown to be a promising technique in overloaded CDMA systems where the number of nodes exceeds the number of spreading sequences [14][15]. One approach to solve this system is the application of the Least Absolute Shrinkage and Selection Operator (LASSO) which is a convex relaxation of the problem stated in (10) to continuous alphabets. For further details the reader is referred to [16]. Other works have proved the feasibility of CS in the M2M context by investigating the delays which occur in CS detection based system where nodes access the channel simultaneously via CDMA [17].

\section{Numerical Evaluation}

\section{A. Classification of Symbol Error Events}

Performing a joint data and activity detection necessitates to distinguish between activity errors and data-symbol errors. So we have to extend the class of errors from simple data errors by considering activity errors, which broadens up classical detection. Estimating an active node to be non-active is generally more severe than the opposite, i.e. estimating a non-active node as active. In the latter case, wrong frames can be detected by error detection mechanisms such as Cyclic Redundancy Check Codes (CRC) which, in contrast, increases the processing cost at higher layers. To distinguish the different error events, we define the following classes for symbol errors:

- Net Symbol errors (NSE)

- False Active errors (FA)

- False Inactive errors (FI)

- Gross Symbol errors (GSE)
We consider the case where a node transmits a data symbol from $\mathcal{A}$ that is detected wrongly as a Net Symbol Error (NSE), additionally, the wrong detection of a symbol from the augmented alphabet $\mathcal{A}_{0}$ is denoted as Gross Symbol Error (GSE). Activity errors are counted when an active node is wrongly estimated to be inactive FI and non-active nodes which are wrongly estimated to be active FA.

Remark: Having the introduced error events in mind, we state that the estimate $\hat{\mathbf{x}}_{i}$ for the problem given in (10) is the MAP estimator on the augmented alphabet $\mathcal{A}_{0}$ which is reflected by the GSE error class. In summary, the MAP detector given in (10) is the optimal detector for minimizing the GSE error event.

\section{B. Classification of Frame Error Events}

For frame wise transmission, we assume the nodes to be active or inactive for the duration of a whole frame containing $L$ symbols. To recover a frame, the detector estimates $L$ consecutive vectors $\hat{\mathbf{x}}_{i}$ with $1 \leq i \leq L$. The frame transmitted by node $k$ is recovered by collecting the $k$ th element in the vectors $\hat{\mathbf{x}}_{i}$ leading to the column vector $\hat{\mathbf{x}}_{k}^{T}=\left[x_{k, 1}, x_{k, 2}, \ldots, x_{k, L}\right]$. The formulation of different error classes on a frame basis requires the definition of a criterion which indicates if a frame is considered to be active or inactive.

$$
\hat{\mathbf{x}}_{k}^{T} \in \begin{cases}\mathcal{A}^{1 \times K} & \text { if criterion is fulfilled } \\ \mathbf{0}_{1 \times K} & \text { if criterion is not fulfilled }\end{cases}
$$

For (13), we point out that an active node can be interpreted as repeating $L$ times the symbol alphabet $\mathcal{A}$ and $\mathrm{x}_{k}$ takes the form

$$
\hat{\mathbf{x}}_{k}^{T}= \begin{cases}{[\mathcal{A}, \mathcal{A}, \ldots, \mathcal{A}] \in \mathcal{A}^{1 \times L}} & \text { if node is active } \\ {[0,0, \ldots, 0] \in \mathbf{0}_{1 \times L}} & \text { if node is non-active }\end{cases}
$$

With (14) we apply a majority criterion and consider an estimated frame as active if $\geq 50 \%$ of the estimated symbols belong to $\mathcal{A}$. Contrary, if $>50 \%$ of the symbols are 0 , the frame is considered to be inactive. Moreover, we define the following three error events on a frame basis:

- Net Frame errors (NFE)

- False Active Frame errors (FA-F)

- False Inactive Frame errors (FI-F)

The Net Frame errors are analogous to NSE, i.e. a frame transmitted by a node containing data symbols from $\mathcal{A}$ that is corrupted after estimation. We consider a frame to be corrupted if at least a single data-symbol is wrong after estimation. False Active Frame errors occur if an inactive node is wrongly estimated to be active on a frame basis and False Inactive Frame errors occur if an active node is wrongly estimated to be inactive on a frame basis. Activity detection on a frame basis is facilitated via the aforementioned majority criterion.

\section{Evaluation of Symbol Errors}

In the following we will discuss the numerical results for the SA-SD. In particular, we investigate the impact of the activity estimation on the overall error rate. To assess the impact of 


\begin{tabular}{|c|c|}
\hline \multicolumn{2}{|c|}{ Simulation Parameters } \\
\hline \hline Number of Nodes & $K=10$ \\
\hline Spreading Gain & $N=16$ \\
\hline Length of Channel Impulse Resp. & $L_{h}=4$ chips \\
\hline Channel Type & Real valued Block Rayleigh Fading \\
\hline Activity Probability & $p_{a}=0.2$ \\
\hline Frame Length & $L=50$ Symbols \\
\hline Modulation Type & BPSK \\
\hline Pre-Whitening & Cholesky Decomposition \\
\hline
\end{tabular}

TABLE I

SimULATION PARAMETER

the joint activity and data detection, we compare our results to a detector that knows which nodes are active and which are not, denoted as Oracle Sphere-Detector. The Oracle SphereDetector will consequently not produce any errors during the activity detection. We first consider the symbol error rates for the scenario summarized in Table I. For pre-whitening we applied the Cholesky Decomposition as derived in Section II-C2. Cholesky Decomposition and Eigenvalue based prewhitening showed absolutely identical performance and we therefore show the results with the application of a Cholesky decomposition based pre-whitening filter only.

Fig. 2 shows the performance of the SA-SD on a symbol basis. As mentioned previously, the regularization term in (10) scales with the noise power which makes the detection of datasymbols from $\mathcal{A}$ costly for low SNR. Consequently, the GSE converge to $p_{a}=0.2$ in the low SNR region. This behavior is obvious since the detector estimates all symbols to zero. The mean number of data symbols from $\mathcal{A}$ contained in $\mathbf{x}_{i}$ is $p_{a}$ and if these symbols are estimated to be zero, $p_{a}$ errors are done on average. This directly influences the NSE which counts data symbols that are estimated wrongly. Obviously, at low SNR all data symbols are mapped to zero resulting in $100 \%$ NSE.

The best possible performance of the SA-SD is achieved if the activity of the nodes is estimated correctly. This bound is shown in Fig. 2 as the Oracle NSE. The Oracle Sphere Detector has knowledge about active and inactive nodes and performs data detection only. In a practical setup this performance can be achieved by complex control signaling about node activity. The performance of the Oracle Sphere Detector is below the NSE rate of the SA-SD revealing that errors during activity detection strongly decrease the NSE rate of the SA-SD.

The error rates for activity detection on a symbol basis are shown on Fig. 3. Considering the FI rate performance clearly shows that especially in the low SNR range the FI rate converges to $100 \%$, i.e. all active nodes are estimated to be non-active. This is again a consequence of the regularization term in (10) which makes symbol detection in a low SNR range costly. In contrast, the FA error rate is very low in a low SNR range, increases to a maximum value of approximately $3 \cdot 10^{-2}$ and decreases again for higher SNR. This behavior results as all nodes are estimated to be non-active at low SNR, consequently, inactive nodes are never estimated to be active.

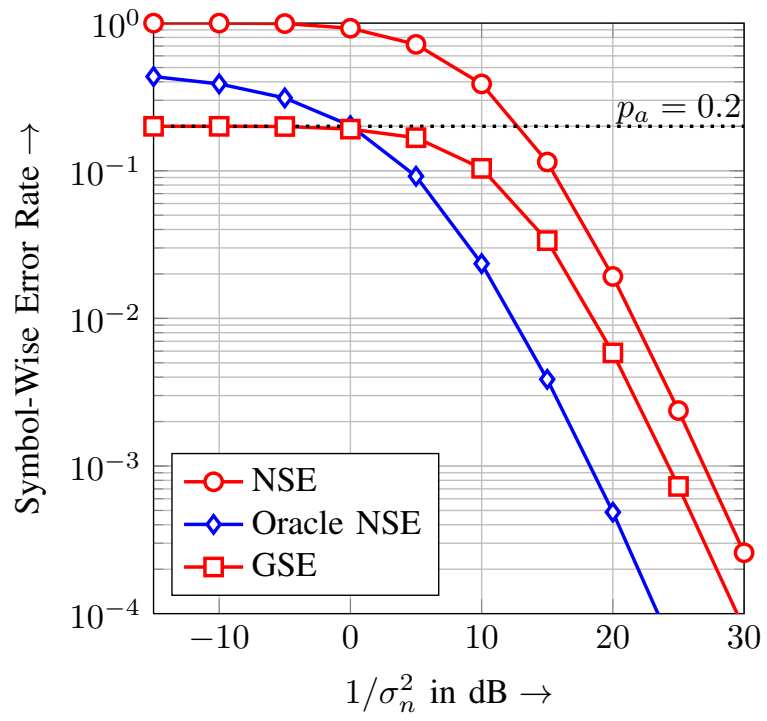

Fig. 2. Net Symbol Errors (NSE) and Gross Symbol Errors (GSE) for Sparsity Aware Sphere Detector compared to an Oracle Sphere Detector

Increasing the SNR, decreases the scaling of the regularization term in (10) due to a lower noise power and some inactive nodes are wrongly estimated to be active. At higher SNR, the corruption of the observation due to noise is decreased and the FA error rate decreases as the SNR increases.

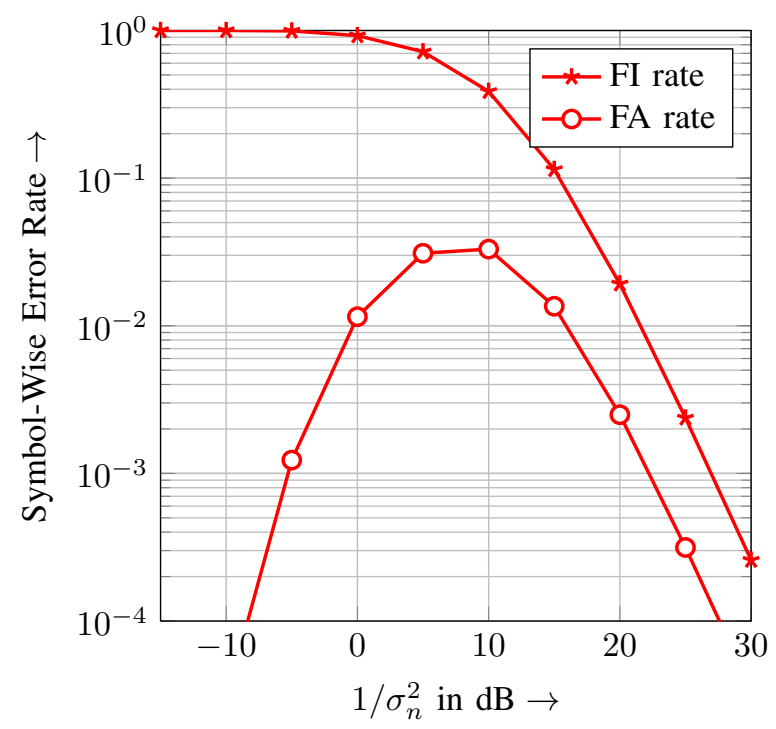

Fig. 3. False Active errors (FA) and False Inactive errors (FI) for the Sparsity Aware Sphere Detector

Figure 4 shows the error rate performance on a frame basis consisting of $L=50$ information symbols. The rate of frames containing information which is corrupted after detection, denoted as NFE, converges to $100 \%$ in the low SNR range and decreases with increasing SNR. This behavior is analogous to the NFE since the detector estimates all symbols to zero for low SNR. Comparing NFE to the NFE errors for 
the Oracle Sphere Detector proves that activity errors again strongly contribute to the NFE error events. Similar to the symbol error events, active nodes are detected by the majority criterion to be non-active on a frame basis. This event is captured by the FI-F curve. Again this curve converges to $100 \%$ at the low SNR range since all symbols are estimated to be zero. As the SNR increases, the FI-F error rate decreases in a similar fashion as the FI error rate decreases on symbol level. During our simulation we were not able to capture a representative rate for the FA-F error rate.

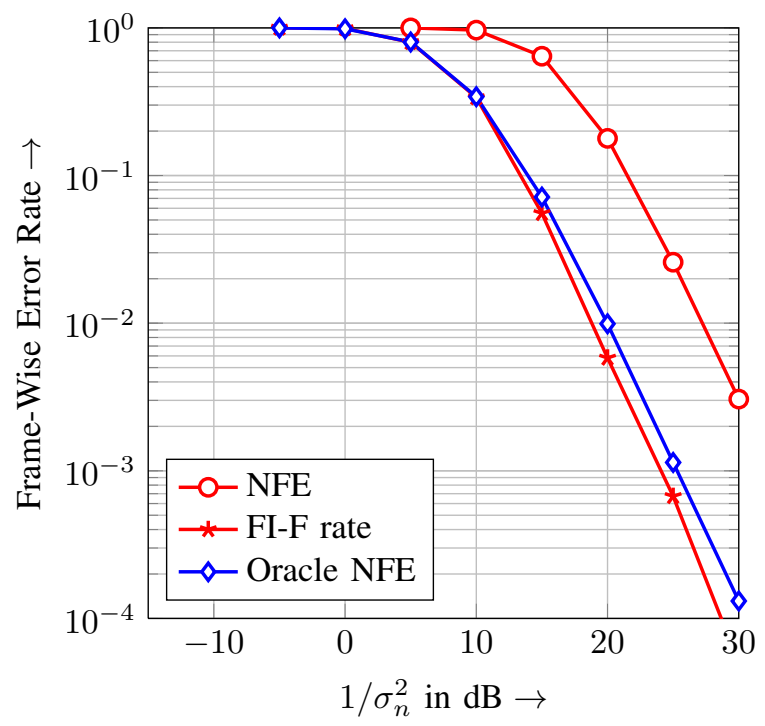

Fig. 4. Net frame errors (NFE) False Inactive Frame errors (FI-F) for Sparsity Aware Sphere Detector compared to an Oracle Sphere Detector

This performance evaluation shows that even though the SA-SD is optimal in the sense of GSE, optimal estimation of node activity is still questionable. As mentioned previously, FA errors can to some extend be identified by higher layers that perform error detection codes such as CRC codes. Whereas FI errors always result in loss of information. This consideration reveals that an optimal detector should also involve higher layer processing capabilities for weighting between the FI and FA errors. This consideration motivates future research on optimal activity and data estimation. Which involves a cross layer design between the physical layer and higher layers.

\section{CONClusion}

In this paper we introduced the application of a Sparsity Aware Multi-User detector on the symbol clock model of a Multi-User CDMA system. We thereby investigated the performance of a Maximum a Posteriori detector to detect node activity and data at symbol rate jointly. We showed that the application of a Cholesky decomposition based pre-whitening filter is efficient in combination with the implementation as Sparsity Aware Sphere Detector. Our investigation revealed that for joint activity and data detection different error classes have to be regarded. We showed that the impact of errors at activity detection are fundamentally different than errors at symbol detection. This consideration motivates to enhance joint activity and data detection by considering cross-layer design approaches.

\section{ACKNOWLEDGMENT}

This work was founded by the German Research Foundation (DFG) under grant DE 759/3-1

\section{REFERENCES}

[1] A. Bartoli, M. Dohler, J. Hernndez-Serrano, A. Kountouris, and D. Barthel, "Low-Power Low-Rate goes Long-Range: the case for secure and cooperative Machine-to-Machine communications," in NETWORKING 2011 Workshops. Berlin, Heidelberg: Springer Berlin Heidelberg, 2011, vol. 6827, pp. 219-230.

[2] S. Lien, K. Chen, and Y. Lin, "Toward ubiquitous massive accesses in 3GPP machine-to-machine communications," Communications Magazine, IEEE, vol. 49, no. 4, pp. 66 -74, Apr. 2011.

[3] EXALTED consortium, "D2.1 - description of baseline reference systems, scenarios, technical requirements \& evaluation methodology," European Collaborative Research FP7 - Project Number: 258512, Tech. Rep., May 2011.

[4] W. Lang, R. Jedermann, D. Mrugala, A. Jabbari, B. Krieg-Brueckner, and K. Schill, "The intelligent container a cognitive sensor network for transport management," IEEE Sensors Journal, vol. 11, no. 3, pp. 688 - 698, Mar. 2011.

[5] S. Verdú, Multiuser Detection. Cambridge, U.K.: Cambridge Univ. Press, November 1998.

[6] V. Kühn, Wireless Communications over Mimo Channels: Applications to CDMA And Multiple Antenna Systems. John Wiley \& Sons, 2006.

[7] P. R. J. J. Rajan, "Model order selection for the singular value decomposition and the discrete karhunen-loeve transform using a bayesian approach," IEEE Proceedings on Vision, Image and Signal Processing, vol. 144 , no. 2, pp. $116-123$, apr 1997.

[8] G. H. Golub and C. F. V. Loan, Matrix Computations, 2nd ed. The Johns Hopkins University Press, 1989.

[9] M. G. Christensen and A. Jakobsson, Multi-Pitch Estimation. Morgan and Claypool Publishers, 2009.

[10] H. Zhu and G. Giannakis, "Exploiting sparse user activity in multiuser detection," IEEE Transactions on Communications, vol. 59, no. 2, pp. 454 - 465, february 2011.

[11] M. O. Damen, H. E. Gamal, and G. Caire, "On maximum-likelihood detection and the search for the closest lattice point," IEEE Transactions on Information Theory, vol. 49, no. 10, pp. 2389 - 2402, oct. 2003.

[12] Y. C. Eldar and G. Kutyniok, Compressed Sensing: Theory and Applications. Cambridge, U.K.: Cambridge Univ. Press, May 2012.

[13] E. J. Candès and M. B. Wakin, "An introduction to compressive sampling," IEEE Signal Processing Magazine, vol. 25, no. 2, pp. 21-30, March 2008.

[14] H. F. Schepker and A. Dekorsy, "Sparse multi-user detection for cdma transmission using greedy algorithms," in 8th International Symposium on Wireless Communication Systems (ISWCS), Aachen, Germany, Nov., pp. $291-295$.

[15] H. Schepker and A. Dekorsy, "Compressive sensing multi-user detection with block-wise orthogonal least squares," in IEEE 75th Vehicular Technology Conference Spring, Yokohama, Japan, May. 2012.

[16] R. Tibshirani, "Regression shrinkage and selection via the lasso," Journal of the Royal Statistical Society, Series B, vol. 58, no. 1, pp. 267-288, 1996.

[17] H. Li, R. Mao, L. Lai, and R. Qiu, "Compressed meter reading for delaysensitive and secure load report in smart grid," in First IEEE International Conference on Smart Grid Communications (SmartGridComm), oct. 2010, pp. $114-119$. 